\title{
When can we use the doubling rule of psa to detect prostate cancer for BPH patients who are treated with 5alpha-reductase inhibitor?
}

\begin{abstract}
Recently, 5alpha-reductase inhibitors (5ARIs) are available for benign prostatic hyperplasia $(\mathrm{BPH})$ patients who have larger size of prostatic volume. 5ARIs reduce the risks of acute urinary retention, surgical operation and prostate cancer. It is well known that the PSA level in those patient decreases to about $50 \%$ of initial level. We use doubling rule of PSA for these patient at the decision making of the biopsy to prostate cancer. But the guidelines of BPH and prostate cancer do not mention when we can use the doubling rule for BPH patients in many countries. Several guidelines provide the information that PSA level reaches to about $50 \%$ after 6 months of treatment. We should know that these data came from the mean value of the patient PSAs. These results suggest that a half of the patient PSAs may not reach to $50 \%$ at that time. If we apply the doubling rule to those patients, the adjusted levels of their PSAs will increase to higher than own initial levels. These data confuse the patients and us. Further study of such as doubling rule or applicable period of the rule will be needed.
\end{abstract}

Volume 5 Issue 5 - 2016

Yoshitatsu Fukabori
Department of Urology, Dokkyo Medical University, Japan

Correspondence: Yoshitatsu Fukabori, Department of Urology, Dokkyo Medical University, 880 Kitakobayashi, Mibu, Shimotsuga, Tochigi 3210293, Japan, Tel 81282872162, Fax 81282867533,Email fukabori@dokkyomed.ac.jp

Received: August 07, 2016 | Published: September 13, 2016

\section{Introduction}

Since finasteride was available for benign prostatic hyperplasia (BPH), the PSA levels of the patients who were treated with 5alphareductase inhibitors (5ARIs) were investigated because of the decrease effect on PSA. ${ }^{1,2}$ Because of the shrinking activity to the large prostate, 5ARIs have been used for reducing the risks of acute urinary retention and surgical operation. How can we screen prostate cancer of these patients? We know the doubling rule of PSA is available for the patients who were treated with 5ARI. But we do not know when this rule is actually and accurately available. The guidelines of BPH and prostate cancer do not provide the answer of this point. This issue is discussed here with some reports.

In European Association of Urology (EAU) guidelines on management of $\mathrm{BPH},{ }^{3}$ there is a description that $5 \mathrm{ARIs}$ act by inducing apoptosis of prostate epithelial cells leading to prostate size reduction of about $18-28 \%$ and circulating PSA levels of about $50 \%$ after $6-12$ months of treatment. The guideline says that the effect of 5ARIs on the serum PSA concentration needs to be considered for prostate cancer screening. When should we begin to use the doubling rule in this long period? There is no information about PSA level after treatment with 5ARIs in American Urological Association (AUA) guideline of BPH. ${ }^{4}$ 'Early detection of prostate cancer AUA guideline' ${ }^{5}$ mentions that finasteride and other $5 \alpha$-reductase inhibitors can decrease PSA values by approximately $50 \%$. However, it is not obvious when the decrease event happens.

There is no information about this phenomenon in 'Early detection of prostate cancer EAU guideline'. ${ }^{6}$ 'NCCN Guideline of prostate cancer early detection" ${ }^{7}$ gives us a caution that medications such as 5ARI (finasteride and dutasteride) are known to decrease PSA by approximately $50 \%$ and PSA values in these men should be corrected accordingly. But, when and how should we do that? These guidelines indicate lots of references about PSA level after treatment with 5ARIs. Twenty years ago, Guess et al. ${ }^{1}$ reported that the serum PSA level should be multiplied by 2 and compared to either age-independent or age-specific upper limits of normal for serum PSA in untreated men with BPH in order to interpret serum PSA levels in men with $\mathrm{BPH}$ treated with finasteride for 6 months or longer. After that, similar proposals $^{2,8}$ to them have been provided because the PSA level after around 6 months from the start of treatment with 5ARIs indicated about $50 \%$ of the initial level in almost all studies of 5ARIs. We easily believed the doubling rule of PSA as standard technique for the patient who was treated with 5ARIs for 20 years. Recently, 5ARIs were commonly used for large size of $\mathrm{BPH}$. We noticed that there were some cases whose PSA levels after application to the doubling rule were higher than their initial levels, especially due to inflammation. ${ }^{9}$

The results may arouse the anxiety of the patients in vain. Why does this strange phenomenon appear? The PSA value is very highly individual. We know that the PSA value of the individual patient is distributed in clinical trials widely. Moreover, large prostate easily suffers some kinds of prostatitis. Prostatitis induces high level of PSA suddenly. The distribution of the PSA does not converge in the small range. We should know that the past result such as $50 \%$ came from the mean value of the patient PSAs. This condition leads us to the suggestion that a half of the patient PSAs may not reach to $50 \%$ after 6 months from the start of 5ARIs. Recently, we examined the PSA value of the BPH patients received 5ARI using Kaplan-Meier method as an endpoint that a PSA value became "approximately $50 \%$ " of initial values. ${ }^{9}$ It took 10 months for a half of the target cases from 5ARI administration to reach "approximately $50 \%$ " of initial PSA. We required 13 months so that $60 \%$ of target cases reached "approximately 50\%" of initial PSA. After 15 months from 5ARI administration, $70 \%$ of target cases reached "approximately $50 \%$ " of initial PSA. The positive predictive value of $50 \%$ should not be recommended. Fifty $\%$ of probabilities are equivalent to expecting to win the front or back of thrown coin. The recommendation degree should exceed at least $50 \%$ for giving some benefit.

The doubling rule may be applicable at least one year after 5ARI administration. Marks et al. ${ }^{10}$ described that the PSA decreases to less than $50 \%$ of initial values $1-3$ years after 5 ARI administration. They recommend that rising up from nadir PSA should be used for decision making of prostate biopsy. 


\section{Conclusion}

It is a matter of course that the adaptation of the biopsy is considered when PSA rose by monitoring of the PSA over time. The patients that we screen prostate cancer may not be always the patients that we know the course of their PSA. The application of the doubling rule to the patient who is treated with 5ARI may sometimes confuse the patients and us. Further study of such as doubling rule or applicable period of the rule will be needed.

\section{Acknowledgments}

None.

\section{Conflicts of interest}

The author declares no conflicts of interest.

\section{Funding}

None.

\section{References}

1. Guess HA, Gormley GJ, Stoner E, et al. The effect of finasteride on prostate specific antigen:review of available data. J Urol. 1996;155(1):3-

2. Roehrborn CG, Boyle P, Nickel JC, et al. Efficacy and safety of a dual inhibitor of 5-alpha-reductase types 1 and 2 (dutasteride) in men with benign prostatic hyperplasia. Urology. 2002;60(3):434-441.
3. Gravas S, Bachmann A, Descazeaud A, et al. Guidelines on the Management of Non-Neurogenic Male Lower Urinary Tract Symptoms (LUTS), incl. Benign Prostatic Obstruction (BPO). European Association of Urology. 2015. p. 1-100.

4. McVary KT, Roehrborn CG, Avins AL, et al. American Urological Association Guideline:Management of Benign Prostatic Hyperplasia (BPH). American Urology Association. 2010. p. 62.

5. Carter HB, Albertsen PC, Barry MJ, et al. Early detection of prostate cancer:AUA guideline. American Urological Association. 2013. p. 28

6. Heidenreich A, Abrahamsson PA, Artibani W, et al. Early detection of prostate cancer: European Association of Urology recommendation. Eur Urol. 2013;64(3):347-354.

7. Carroll PR, Parsons JK, Andriole G, et al. NCCN Guidelines Insights: Prostate Cancer Early Detection. J Natl Compr Canc Netw. 2016;14(5):509-519.

8. Tsukamoto T, Endo Y, Narita M. Efficacy and safety of dutasteride in Japanese men with benign prostatic hyperplasia. Int $J$ Urol. 2009;16(9):745-750.

9. Fukabori Y, Yashi M, Betsunoh H. PSA value after administration of dutasteride. Preventive Nephrology and Urology. 2016;24(1):61-63.

10. Marks LS, Andriole GL, Fitzpatrick JM, et al. The interpretation of serum prostate specific antigen in men receiving 5alpha-reductase inhibitors:a review and clinical recommendations. J Urol. 2006;176(3):868-874. 